REVUE FRANCOPHONE DE LA

DÉFICIENCE INTELLECTUELLE

VOLUME 29, 19-35

\title{
LE PLAN D'INTERVENTION EN CONTEXTE D'INTERVENTION COMPORTEMENTALE INTENSIVE (ICI) : PERCEPTION DES PRATIQUES ET DES DÉFIS
}

\begin{abstract}
Annie Paquet ${ }^{1}$, Carmen Dionne ${ }^{2}$ et Myriam Rousseau ${ }^{3}$.
Résumé : La mise en œuvre de l'intervention comportementale intensive (ICI) nécessite une planification des objectifs et des moyens pour chaque enfant. Ce processus d'individualisation est essentiel à une intervention de qualité. Selon la loi, les établissements doivent rendre compte de la planification des interventions pour chacune des personnes à qui ils offrent des services par le biais du plan d'intervention. Cette étude vise à identifier les pratiques et les défis associés à l'élaboration de ces plans, en contexte d'ICI, du point de vue de membres de l'équipe d'intervention (21 intervenantes, 6 superviseures et 4 professionnelles). L'analyse des entretiens semi-structurés permet de préciser les pratiques et les défis perçus, notamment ceux associés aux caractéristiques de l'ICI (p. ex., son intensité), à la participation des parents dans le processus, au travail interdisciplinaire, à l'individualisation, à l'accès aux données probantes venant appuyer la planification de l'intervention et aux normes de l'établissement.
\end{abstract}

Mots clés : Intervention comportementale intensive, autisme, plan d'intervention, perception des intervenants.

\section{Introduction}

L'intervention comportementale intensive (ICI) a fait l'objet d'un grand nombre d'études, de recensions systématiques et de méta-analyses (Eldevik et al., 2010; Kasari et Lawton, 2010; Kuppens et Ongena, 2012; Makrygianni et Reed, 2010; Makrygianni, Gena, Galanis et Katoudi, 2018; Odom, Collet-Klingenberg, Rogers et Hatton, 2010; Prior, Roberts, Rodger, Williams et Sutherland, 2011; Reichow, Barton, Boyd et Hume, 2012; Virués-Ortega, 2010; Warren et al., 2011; Weitlauf et al., 2014). Plusieurs guides et avis recommandent son utilisation pour les jeunes enfants ayant un trouble du spectre de l'autisme (TSA), tant au Québec qu'à l'international (Haute Autorité de santé, 2012; Institut national d'excellence en santé et en services sociaux [INESSS], 2014; Weitlauf et al., 2014). L'ICI est une intervention qui se veut précoce, puisqu'offerte en bas âge (avant 6 ans), et intensive, puisqu'elle est appliquée entre 20 et 40 heures par semaine (Reichow, Hume, Barton et Boyd, 2018). Au Québec, l'intensité se traduit davantage par une intervention dont le dosage est de près d'une

1- Annie Paquet, Ph.D., Professeure à l'Université du Québec à TroisRivières (UQTR) au département de psychoéducation. Toute correspondance en lien avec cet article devrait parvenir à annie.paquet@uqtr.ca. 2 - Carmen Dionne, Ph.D., Professeure à l'UQTR au département de psychoéducation. 3 - Myriam Rousseau, Ph.D., chercheuse en établissement à l'Institut universitaire un DI-TSA rattachée au Centre intégré universitaire de santé et de services sociaux de la Mauricie-et-du-Centre-du-Québec. vingtaine d'heures par semaine (INESSS, 2014; ministère de la Santé et des Services sociaux [MSSS], 2003). Elle prend appui sur les principes de l'analyse appliquée du comportement (AAC), bien que plusieurs établissements rapportent y intégrer également l'approche développementale (Dionne, Joly, Paquet, Rousseau et Rivard, 2016). L'AAC peut être décrite comme une discipline appliquée de l'analyse du comportement, prenant appui sur le behaviorisme, donc sur l'analyse du comportement et de ses relations avec l'environnement (pour la description formelle de cette discipline, voir Baer, Wolf et Risley, 1968). L'intervention vise à soutenir le développement de l'enfant de façon globale (habiletés de communication, de jeu, sociales, cognitives, d'autonomie), en mettant l'emphase sur les habiletés qui sont fonctionnelles et en incluant les sphères plus caractéristiques de l'autisme (Klintwall et Eikeseth, 2014).

Il existe différents modèles d'ICI. Ces modèles peuvent se distinguer sur plusieurs composantes, telles que : l'âge des participants, le dosage de l'intervention, les environnements dans lesquels l'intervention est offerte, les personnes qui implantent (parents, intervenants) ainsi que le protocole privilégié (incluant la place de la famille dans les différentes étapes du processus d'intervention, les outils d'évaluation et les procédures d'intervention) (Långh, Cauvet, Hammar et Bölte, 2017). Il faut dire que dans le cadre de l'ICI au 
Québec, les personnes qui mettent en place l'intervention sont majoritairement des techniciens en éducation spécialisée, qui sont supervisés par des professionnels, principalement des psychoéducateurs ou spécialistes en activité clinique, auxquels peuvent se joindre des orthophonistes et des ergothérapeutes (Paquet, Dionne, Joly, Rousseau et Rivard, 2017). Odom, Boyd et Hume (2010) ont répertorié 30 modèles globaux d'intervention, dont celui du Lovaas Institute, mais également d'autres modèles tels que Learning experiences: An alternative program for preschoolers and parents (LEAP; Strain et Bovey, 2011), le Project DATA (Developmentally Appropriate Treatment for Autism; Schwartz, Ashmun, McBride, Scott et Sandall, 2017; Schwartz, Sandall, McBride et Boulware, 2004) ou le Walden Model (McGee, Morrier et Daly, 1999). Le modèle d'intervention sert donc à préciser les grandes dimensions de l'intervention à offrir. Au-delà de ces grandes dimensions, il est nécessaire de planifier de façon minutieuse les cibles et les stratégies d'intervention à offrir à chacun des enfants. Cela signifie que les équipes cliniques doivent préciser les objectifs poursuivis et les moyens spécifiques à mettre en place pour un enfant, et ce, en considérant les orientations générales du modèle d'ICI retenu.

Afin de rendre compte des choix réalisés, les personnes qui mettent en place des interventions doivent décrire le processus de planification à l'intérieur d'un document officiel, le plan d'intervention (PI). Le PI a pour fonction : 1) la planification de l'intervention; 2) la gestion de l'intervention; 3) la concertation entre l'équipe d'intervention et l'enfant et sa famille; 4) la communication entre les différents acteurs concernés; et 5) le partage des responsabilités (Goupil, 2004). Tel que défini à l'article 102 de la loi sur les services de santé et les services sociaux (MSSS, 1972), un PI doit être élaboré pour chacune des personnes recevant des services d'établissements soumis à cette loi. La loi prévoit que ce plan précise, en collaboration avec la personne ou ses représentants, les besoins, les objectifs poursuivis, les moyens à mettre en place ainsi que la durée prévue des services. Il est également prévu que ce plan soit révisé sur une base régulière afin d'apporter les ajustements nécessaires. Cet article précise aussi que le PI doit permettre de coordonner les services dispensés par les différents intervenants concernés de l'établissement. De plus, les établissements du réseau de la santé possèdent leur propre processus clinique offrant des balises à l'intervenant relativement aux principes généraux du processus d'identification des objectifs et des moyens à mettre en place (p. ex., Centre de réadaptation en déficience intellectuelle et en trouble envahissant du développement de la Mauricie et du Centre-du-Québec Institut universitaire [CRDITED MCQ - IU], 2013). Ce processus est généralement le même pour un ensemble de services. S'y retrouvent des directives concernant notamment les délais à respecter pour l'élaboration et la révision des objectifs, la participation de la personne et de ses proches, ainsi que la forme que doit prendre le PI.

En fait, l'individualisation de l'intervention est centrale en ICI (Leaf et al., 2016; Leaf et McEachin, 2016). Leaf et McEachin (2016) précisent qu'une ICI de qualité nécessite que les décisions cliniques reposent sur une observation attentive des besoins changeants de chacun des enfants et de leur réponse unique à l'intervention proposée. En effet, la variété des profils des enfants ayant un TSA, qui est désormais bien documentée (Masi, DeMayo, Glozier et Guastella, 2017), renvoie à la nécessaire adaptation de l'intervention. À cet effet, une étude récente montre cette diversité des profils des enfants fréquentant les établissements québécois, malgré qu'une majorité d'entre eux présentent des profils déficitaires dans plusieurs sphères de leur développement (Dionne et al., 2016). Une bonne compréhension du développement de l'enfant, de ses caractéristiques et de ses besoins permet d'identifier les objectifs d'intervention (Klintwall et Eikeseth, 2014; Leaf et McEachin, 2016; Rousseau, Dionne et Paquet, 2012). En plus du profil individuel de l'enfant, les réalités familiales et les différents contextes dans lesquels l'enfant évolue sont susceptibles d'influencer le choix des objectifs (Leaf et McEachin, 2016; McCollow et Hoffman, 2018). D'ailleurs, en intervention précoce, la participation active des parents dans le cadre du processus d'élaboration de l'intervention est recommandée (Division for Early Childhood [DEC], 2014; McCollow et Hoffman, 2018; Strauss et al., 2015).

Une fois les objectifs ciblés, les intervenants ont à sélectionner les stratégies d'intervention à mettre en place pour les atteindre. Il faut savoir qu'à l'intérieur d'un même modèle global d'intervention, plusieurs stratégies spécifiques peuvent être utilisées (Odom et al., 2010). En effet, les intervenants sont appelés à sélectionner plusieurs stratégies spécifiques d'intervention afin de répondre aux besoins de chacun des enfants (Dingfelder et Mandell, 2011). La sélection des stratégies d'intervention les plus appropriées est une étape cruciale $\mathrm{du}$ processus clinique (McCollow et Hoffman, 2018; Romanczyk et Gillis, 2011). De plus, en cours 
d'intervention, le jugement clinique permettant d'ajuster les stratégies reposera sur une multitude de facteurs, notamment le comportement, la motivation, les performances de l'enfant dans le passé, son niveau d'activité (agité/calme), son niveau d'attention, sa réceptivité à l'intervention, les comportements qui interfèrent avec l'apprentissage et leurs fonctions (Leaf et McEachin, 2016). D'ailleurs, l'évaluation de l'atteinte des objectifs ciblés, c'est-à-dire des progrès de l'enfant, est un des éléments caractéristiques de l'ICI (Reichow et al., 2018). Cette évaluation nécessite donc l'établissement d'objectifs observables et mesurables, de même qu'une description des stratégies à mettre en place afin d'en assurer un suivi étroit.

Des auteurs rappellent la complexité de la planification de l'intervention et donc, le besoin de formation des personnes qui appliquent l'intervention (Leaf et al., 2016). En effet, celles-ci doivent pouvoir disposer d'une bonne connaissance des données probantes, mais surtout une bonne connaissance des principes qui servent d'assises aux interventions. Or, l'instabilité des équipes et le manque de formation en autisme (Gamache, Joly et Dionne, 2011) sont notamment évoqués, parmi les défis d'implantation de l'ICI dans des établissements québécois

L'élaboration d'un PI pour les jeunes enfants ayant un TSA est donc un processus complexe et multidimensionnel, qui tient compte d'un ensemble de facteurs (Tarbox et al., 2013). Cependant, à notre connaissance, peu d'informations sont disponibles quant aux défis rencontrés relativement à l'élaboration de PI par des établissements du réseau en contexte d'ICI.

\section{Objectif}

Cette étude s'inscrit dans le cadre d'un plus vaste projet de recherche visant à obtenir des informations concernant les pratiques et les perceptions des intervenants au regard de la planification de l'intervention en contexte d'ICI. Plus précisément, cet article traitera du premier volet de cette étude dont l'objectif principal est d'identifier et de mieux comprendre les défis associés à l'élaboration des PI dans le contexte de l'ICI, et ce, du point de vue des membres de l'équipe d'intervention qui y participent.

${ }^{1}$ Il faut préciser qu'une ancienne superviseure, désormais psychoéducatrice au sein de la direction de la qualité, de la recherche, du développement et de l'innovation, a également participé en se référant à son expérience antérieure.

\section{Méthode}

Une méthode qualitative de recherche, par le biais d'entretiens semi-dirigés individuels, a été privilégiée. En effet, cette méthode de collecte de données permet d'avoir accès au regard des intervenantes sur leur propre travail. Elle permet d'approfondir le point de vue des répondantes, de comprendre la situation selon leur perspective (Boutin, 2018).

\section{Participants et recrutement}

Le projet s'est déroulé dans un établissement du réseau de la santé et des services sociaux du Québec, plus spécifiquement au sein du programme services DI-TSADP. Les participants devaient être à l'emploi de l'établissement dans le cadre du programme ICI depuis au moins six mois et avoir participé à l'élaboration ainsi qu'à la révision d'au moins un PI. Trois principales catégories de répondantes participent à l'étude 1) les techniciennes en éducation spécialisée (TES), responsables du PI des enfants au sein du programme ICI, 2) les superviseures, toutes psychoéducatrices, qui offrent du soutien à ces techniciennes ${ }^{1}$, et 3 ) les professionnelles (ergothérapeute, orthophoniste ou agent de programmation et de planification de la recherche [APPR]) qui agissent dans le cadre du programme ICI. Ainsi, ce sont 21 TES, 5 superviseures actuelles et 1 ancienne superviseure et 4 professionnelles qui ont participé aux entretiens. Elles étaient réparties dans différents points de services sur le territoire que dessert l'établissement. Ces participantes représentent 52,5\% des TES, 83,3\% des superviseures (auquel s'ajoute une ancienne superviseure) et $80 \%$ des professionnelles du programme d'ICI sur le territoire de l'établissement.

Années d'expérience. En moyenne, les répondantes possèdent 8,42 années d'expérience $(E ́ T=4,07$; $\min : 1$; $\max : 15)$ auprès des enfants ayant un TSA. De façon plus spécifique, elles possèdent 5,97 années d'expérience (ÉT $=3,52 ; \min : 0,5 ; \max : 14$ ) dans le cadre du programme d'ICI (voir Tableau 1). Il faut souligner que les établissements de santé et de services sociaux ont reçu le mandat d'implanter l'ICI en 2003, il y a donc 12 ans. 
Tableau 1

Nombre d'années d'expérience des participants $(N=31)$

\begin{tabular}{|c|c|c|c|c|c|c|c|c|}
\hline \multirow[b]{2}{*}{ Groupe } & \multicolumn{4}{|c|}{ Client TSA } & \multicolumn{4}{|c|}{ Programme ICI } \\
\hline & $M$ & $E ́ T$ & Min & $\operatorname{Max}$ & $M$ & $\dot{E} T$ & Min & Max \\
\hline $\begin{array}{l}\text { TES } \\
(n=21)\end{array}$ & 8,09 & 3,77 & 1 & 14 & 6,31 & 3,62 & 0,5 & 12 \\
\hline Superviseures $(n=6)$ & 10,83 & 4,26 & 5 & 15 & 6,67 & 3,67 & 3 & 12 \\
\hline $\begin{array}{l}\text { Professionnelles } \\
(n=4)\end{array}$ & 5,67 & 4,85 & 1,5 & 12 & 3,13 & 1,11 & 1,5 & 4 \\
\hline Total & 8,42 & 4,07 & 1 & 15 & 5,97 & 3,52 & 0,5 & 12 \\
\hline
\end{tabular}

$\overline{\text { Note. }} \mathrm{TSA}=$ Trouble du spectre de l'autisme; TES=Technicien en éducation spécialisée; ICI= Intervention comportementale intensive.

Formation. Toutes catégories confondues, vingt-etune participantes suivent ou ont suivi une formation universitaire en TSA. Pour l'ensemble d'entre elles, il s'agit d'une formation offerte dans le cadre d'un programme national de formation. Ce sont 16 de ces 21 participantes qui avaient complété cette formation lors de l'entrevue, alors que les 5 autres étaient en cours de formation.

Les TES sont détentrices, en majorité, d'un diplôme d'études collégiales $(n=14 / 21)$, bien qu'une partie d'entre elles possèdent un diplôme universitaire (6/21). Il faut noter ici qu'il s'agit du dernier diplôme obtenu avant l'obtention du certificat dans le cadre d'un programme national de formation. L'information est manquante pour une des intervenantes. Les superviseures, quant à elles, sont toutes détentrices d'un diplôme d'études universitaire donnant accès à l'Ordre des psychoéducateurs et psychoéducatrices du Québec. Les professionnelles détiennent également toutes des diplômes universitaires donnant accès à un ordre professionnel.

Nombre de plans d'intervention annuellement. Lorsqu'interrogées quant au nombre de PI auxquels elles participent annuellement, la grande majorité des TES répondent un seul (16/21), alors que certaines répondent deux (4/21). La donnée est manquante pour l'une des répondantes. En ce qui concerne les superviseures, le nombre de PI auxquels elles participent annuellement varie entre 6 et 16. La donnée est manquante pour l'une des répondantes. Pour les professionnelles, à l'exception de l'APPR, le nombre varie de 10 à 30 .

\section{Instrument}

Un guide d'entretien a été élaboré aux fins de l'étude. Il inclut trois grands volets associés aux pratiques d'élaboration des PI et des programmations individuelles en contexte d'ICI, soit 1) les défis associés à l'élaboration des PI (p. ex., Selon vous, quels sont les défis associés à l'élaboration des PI et des programmations individualisées en contexte d'ICI), 2) les besoins de soutien des intervenants (p. ex., Selon vous, de quoi avezvous besoin afin de faciliter le processus d'élaboration des PI ?), ainsi que 3) les solutions et les innovations provenant du milieu (p. ex., Avez-vous des solutions à proposer concernant les défis d'utilisation des PI ou des plans de leçon en contexte d'ICI ? Avez-vous développé des outils maison, des stratégies afin de faciliter le processus ?). Des informations sociodémographiques sont également recueillies auprès des répondantes en début d'entretien (formation, expérience, nombre de plans d'intervention auxquels elles contribuent par année).

\section{Procédures}

La chercheuse principale et l'assistante de recherche ont communiqué directement, par le biais de courriels ou d'appels téléphoniques, avec les intervenantes ayant consenti à participer. Ils convenaient alors d'un moment pour réaliser un entretien et également de la modalité privilégiée (téléphonique ou en personne). Les entretiens ont été réalisés en majorité en personne dans les locaux de l'établissement. Ils étaient d'une durée maximale de 60 minutes dans un souci d'éviter de surcharger les répondantes. Ils ont été enregistrés de façon audio et 
retranscrits dans leur intégralité aux fins d'analyses. Ils sont réalisés pendant le mois de mai 2015.

Le comité d'éthique de la recherche de l'Université du Québec à Trois-Rivières, de même que le comité d'éthique conjoint de la recherche des centres de réadaptation en déficience intellectuelle et en troubles du spectre de l'autisme ont approuvé la réalisation du projet.

\begin{abstract}
Analyses
Des analyses qualitatives de contenu ont été réalisées à partir de catégories préétablies avec ouverture sur des catégories émergentes (Paillé et Mucchielli, 2016). Plus précisément, le verbatim des entretiens est retranscrit par les assistants de recherche. Par la suite, une relecture attentive de l'ensemble des entretiens est faite par la chercheuse afin de se familiariser avec le contenu. Une catégorisation est réalisée à partir des unités de sens dégagées. Des thèmes émergents sont retenus et classés dans les grandes rubriques ayant servi à élaborer de guide d'entretien semi-dirigé. Les informations sociodémographiques ont fait l'objet d'une analyse descriptive.
\end{abstract}

\section{Résultats}

Les résultats sont présentés selon les rubriques suivantes : 1) les caractéristiques du programme ICI; 2) la participation parentale; 3) le travail interdisciplinaire; 4) l'individualisation de l'intervention; 5) les bonnes pratiques et les données probantes; ainsi que 6) le processus clinique mis en place par l'établissement. Pour chacune des rubriques sont abordées les perceptions des répondantes relatives aux défis d'élaboration des PI en contexte d'ICI, ainsi que les solutions et innovations proposées ou les besoins de soutien des intervenantes, qui correspondent aux grandes rubriques du guide d'entretien.

\section{Les caractéristiques du programme ICI}

L'analyse de contenu réalisée permet de relever que différents aspects du programme ICI peuvent contribuer aux défis d'élaboration des PI. En effet, il est possible de regrouper les propos des répondantes relativement à ces défis et la façon dont ils influencent l'élaboration des PI en cinq principales catégories: 1) le grand nombre d'objectifs; 2) le lieu d'intervention; 3) les cotations; 4) le degré de familiarisation avec l'ICI; et 5) les curricula utilisés.

Le grand nombre d'objectifs. La planification d'un grand nombre d'objectifs, associé à l'intensité du programme, peut constituer un défi. Les intervenantes évoquent l'importance de bien planifier le temps qui leur est imparti. La transmission de l'information concernant ce grand nombre d'objectifs de travail auprès de la famille de l'enfant est considérée comme un défi par une répondante. Les intervenants rapportent qu'habituellement les PI contiennent un nombre relativement restreint d'objectifs. Cette volonté d'avoir recours à un petit nombre d'objectifs repose notamment sur le désir de rendre cet outil accessible et convivial pour les parents. Selon les propos recueillis, il peut donc être difficile de trouver un équilibre entre un nombre raisonnable d'objectifs à inscrire au PI et le grand nombre d'habiletés à développer chez l'enfant.

Le lieu d'intervention. L'ICI peut se dérouler dans différents environnements. Pour plusieurs enfants, l'intervention se déroule, du moins en partie, en milieu de garde. Pour d'autres, l'intervention est réalisée à domicile. Des répondantes rapportent des défis quant à l'ajustement de l'intervention en fonction des caractéristiques des milieux. L'environnement dans lequel travaillent les intervenantes peut venir teinter le choix des stratégies mises en place. En effet, les milieux accueillant l'enfant ont parfois des valeurs ou une conception de l'intervention qui est différente de celle mise de l'avant en contexte d'ICI.

"Si on parle de défis, il y a aussi au niveau des interventions, c'est de composer avec l'environnement [...] Par exemple si tu es dans une garderie bien faut savoir, adapter nos interventions selon l'endroit où on intervient puis, selon les possibilités qu'on a pour pouvoir intervenir... Puis tenir compte des valeurs de tout le monde... ». (Participant TES 01)

Les protocoles. Il existe différents protocoles en ICI, qui sont associés à des modèles d'intervention (Leaf et McEachin, 1999; Maurice, Green et Luce, 1996). Ces protocoles fournissent notamment des informations relatives aux cibles d'interventions et aux moyens d'intervention à privilégier. Les répondantes mentionnent avoir de la difficulté à avoir accès à des protocoles autres que celui de Lovaas (1996). D'ailleurs, des répondantes soulignent que la programmation demeure parfois trop collée sur ce protocole, ce qui peut limiter le choix des interventions à privilégier auprès des enfants et qui résulterait en un manque d'individualisation.

"Les éducatrices aiment beaucoup [le protocole de] Catherine Maurice, vraiment, vraiment, beaucoup et parfois, je trouve que 
ça fait trop des recettes rigides aussi làdedans [...] On dirait que parfois, c'est trop établi dans une recette. Je comprends que c'est sécurisant être là-dedans, j'aimerais beaucoup ça moi aussi avoir des recettes, mais je n'en ai pas... ». (Participant PROF 01)

La connaissance du programme. Les répondantes soulignent les disparités en ce qui concerne le niveau de connaissance du programme au sein de l'équipe d'intervention. Tout le personnel n'est pas formé aux approches et aux stratégies utilisées dans le programme ICI. Ils n'ont pas tous recours aux mêmes techniques. D'ailleurs, l'analyse relève des défis associés au manque d'uniformisation du vocabulaire spécifique aux approches et stratégies d'intervention. Ce niveau de connaissance quant aux stratégies d'intervention peut influencer le choix des moyens identifiés au PI.

Pour faire face aux défis. Pour certaines, la programmation individuelle, complémentaire au PI et, bien souvent, intégrée à celui-ci en annexe, permet de répondre au défi du grand nombre d'objectifs visés en raison de l'intensité. La programmation individuelle est un document plus détaillé que le PI et qui permet de rapporter l'ensemble des habiletés qui sont enseignées à l'enfant, des stratégies d'intervention à mettre en place, de même qu'à la prise de mesure permettant le suivi des progrès. En effet, selon les répondantes, la programmation individualisée permet l'identification d'un plus grand nombre de cibles d'intervention et des ajustements plus fréquents que ce qui est possible dans le cadre d'un processus formel de PI. Les objectifs y sont microgradués. Une répondante souligne d'ailleurs le besoin de rendre les liens entre le PI et la programmation plus explicites afin d'en faciliter la compréhension par le parent.

Des besoins d'informations sont rapportés. Les répondantes aimeraient pouvoir avoir accès à un répertoire d'informations afin de connaitre les différents modèles et protocoles disponibles, ainsi que pour obtenir de l'information sur les approches, méthodes ou procédures d'intervention à utiliser en ICI, et ce, en français. Certaines répondantes aimeraient pouvoir disposer de protocoles plus adaptés aux enfants présentant de plus grands écarts à la moyenne en termes de développement.

Dans un autre ordre d'idées, il est proposé de créer un lieu de partage afin d'échanger sur les pratiques d'élaboration de la programmation de chacun. Ce serait l'occasion de relever les avantages des différentes procédures et d'harmoniser les pratiques.

\section{La participation parentale en contexte de planification de l'intervention en ICI}

Malgré la reconnaissance de l'importance de la participation des parents lors de l'élaboration du PI, bon nombre de répondantes soulèvent des défis auxquels elles doivent faire face dans leur quotidien afin de favoriser la participation des parents aux différentes étapes du processus d'élaboration. En effet, elles identifient les défis suivants: 1) le soutien aux parents dans la formulation de leurs besoins prioritaires, 2) le soutien à la participation des parents lors de la planification de l'intervention, 3) la participation des parents dans la planification et l'application des moyens et 4) les disponibilités pour les rencontres.

Le soutien aux parents dans la formulation de leurs besoins prioritaires. Dans le cadre du processus d'élaboration du PI, les intervenantes rapportent qu'elles sont appelées à soutenir les parents dans la formulation de leurs besoins. Pour certaines, cet exercice s'avère délicat et peut confronter l'intervenante dans ses valeurs et ses perceptions des priorités d'intervention auprès de l'enfant. En effet, il est question d'une recherche d'équilibre entre les besoins nommés par les parents et les besoins identifiés par l'équipe d'intervention, notamment dans le cadre de l'évaluation initiale. Selon certaines répondantes, il arrive que les points de vue respectifs ne concordent pas. Cette étape de la planification exige donc de l'intervenante plusieurs habiletés qui diffèrent de leur travail au quotidien avec l'enfant, tel que de mener des entrevues afin de permettre aux parents de réfléchir à leurs propres besoins et de les nommer. De plus, l'intervenante doit être attentive à ce que le parent essaie de lui transmettre et valider auprès de ce dernier s'il saisit bien ses propos. Pour certains parents, l'identification des besoins peut constituer un défi. Aussi, les besoins que rapportent les parents en début de services, donc lors de l'élaboration du PI, sont appelés à changer avec le temps, pouvant nécessiter des ajustements réguliers.

Le soutien à la participation de la famille lors de la planification de l'intervention. Le soutien à la participation parentale dans le cadre de l'élaboration des PI en contexte d'ICI nécessite que les intervenantes se sentent à l'aise avec les familles et qu'elles développent des compétences relatives au travail auprès de celles-ci ( $p$. ex., la capacité à mener des entretiens avec les parents, à établir une relation de confiance, à favoriser la 
participation). Pour certaines, cela constitue un défi, les intervenants étant plus à l'aise avec le travail quotidien auprès de l'enfant plutôt qu'avec les parents. Les répondantes mentionnent d'ailleurs qu'il peut être complexe pour les parents de comprendre tout le processus clinique, sa raison d'être et sa pertinence. Les visites à domicile sont l'occasion de s'assurer que les parents ont une bonne compréhension des objectifs et des moyens précisés au PI. Afin de les soutenir dans cette compréhension, certaines répondantes rappellent l'importance d'éviter d'utiliser le jargon professionnel.

Les répondantes soulignent que certains parents sont moins disponibles en raison notamment de l'adaptation nécessaire suite à l'annonce d'un diagnostic de TSA pour leur enfant. Dans ce contexte, l'intervenante est appelée à accompagner le parent, notamment en transmettant de l'information sur l'autisme, sur les interventions. Selon les répondantes, ces étapes sont nécessaires à la collaboration. Cependant, cette forme de soutien ne serait souvent pas clairement indiquée au PI.

\begin{abstract}
"Je trouve qu'on a un mandat de les former, informer, mais cela n'apparait pas nulle part, puis des fois je me dis si cela pouvait être consigné d'une certaine façon cela nous rappelle aussi, d'intervenir là-dessus.» (Participant SUP 02)
\end{abstract}

La participation des parents dans la planification et la mise en place des moyens. Bien que l'importance de la participation du parent en contexte d'ICI soit mentionnée par plusieurs, certains obstacles semblent freiner celle-ci. Notamment, lors de l'élaboration du PI, un défi technique est rapporté en lien avec le système informatisé. En effet, le formulaire du PI ne comporterait pas de section réservée à la participation des parents. Néanmoins, certaines répondantes notent au PI l'implication de chacun relativement aux moyens à mettre en place. Cette implication parentale devrait d'ailleurs, selon certaines, être encore plus mise de l'avant, notamment lors de la planification de la généralisation des apprentissages chez l'enfant. Pour les répondantes, un suivi étroit des interventions doit être fait avec les parents, surtout lors de la révision des programmations. Il semble pourtant que ce partage d'informations sur la programmation quotidienne de l'enfant puisse constituer un défi en soi, surtout lorsque l'intervention ne se déroule pas au domicile. L'une des répondantes rappelle l'importance que la famille puisse bien comprendre les différents apprentissages et moyens ciblés au PI de l'enfant. Elle souligne l'importance de bien expliciter les interventions retenues, de soutenir les parents afin qu'ils puissent utiliser certains des moyens dans leur quotidien.

"J'essaie d'aller au moins une fois par
semaine dans la famille pour pouvoir
généraliser tout cela, le présenter à la famille,
pour pouvoir les voir en action aussi et qu'ils
puissent, euh, se mettre les mains à la pâte
aussi et de voir les retombées de tout cela."
(Participant TES 18)

Les répondantes mentionnent également que la participation des parents sur le plan de l'évaluation des apprentissages peut constituer un défi, notamment lors de la complétion des grilles d'observation.

\section{"Le parent, il faut vraiment qu'il soit motivé à remplir les grilles soit par un objectif à la maison... il faut les motiver beaucoup à le faire, puis en rappeler l'importance." (Participant TES 22)}

Disponibilités pour les rencontres. Au-delà des défis de soutien à la participation des familles dans l'élaboration du PI ou aux différentes étapes du processus clinique, des répondantes mentionnent qu'il est difficile de fixer des rencontres, ce qui peut avoir un impact sur le respect des délais accordés pour l'élaboration du PI.

Pour faire face aux défis. Des répondantes proposent de créer des documents simples, destinés aux parents, afin de leur permettre de mieux comprendre les services (dont le PI), le rôle des différents intervenants et leur implication possible dans le processus. Une répondante propose d'ailleurs d'accroître l'offre de formations offertes aux familles sur l'intervention auprès des jeunes enfants autistes afin notamment de favoriser une meilleure compréhension des outils et des moyens utilisés. Certaines répondantes soulignent la contribution possible de partenaires, notamment les intervenants sociaux. La présence de ces intervenants est perçue comme un soutien possible à la participation du parent lors du premier PI.

Dans une perspective un peu plus technique, certaines soulignent des besoins d'adaptation du système informatisé qui supporte le PI. En effet, afin de rendre le PI plus fonctionnel et aussi pour préciser le rôle de l'ensemble des personnes concernées, il a été proposé de permettre d'inclure les parents ou les éducateurs du milieu de garde comme responsables de la mise en place d'un moyen pour un objectif inscrit au PI. 
Le travail en interdisciplinarité en contexte de planification de l'intervention en ICI

L'analyse de contenu réalisée permet de mettre en lumière différents thèmes associés aux défis du travail interdisciplinaire en contexte de planification des interventions en ICI. En effet, il est possible de regrouper les propos des répondantes en cinq principales catégories : 1) la familiarisation avec l'articulation du processus clinique en équipe interdisciplinaire; 2) l'accessibilité aux professionnels; 3) la multiplication du nombre de personnes présentes lors de la rencontre de planification du PI; 4) les disponibilités de l'équipe de travail pour les rencontres conjointes; et 5) les difficultés rencontrées par la TES quant à la mise en place, le suivi et l'évaluation de certains objectifs plus spécifiques à une discipline.

La familiarisation avec l'articulation du processus clinique en équipe interdisciplinaire. L'une des répondantes rappelle que les intervenants et professionnels doivent se familiariser avec le processus clinique tel qu'il s'articule en contexte de travail interdisciplinaire. En effet, certains aspects de l'organisation du processus clinique diffèrent dans le cadre du travail interdisciplinaire, soit le nombre de personnes impliquées dans l'évaluation, la procédure de collecte de données ainsi que la planification et la révision du PI. Il est notamment nécessaire de définir les rôles de chacun au sein de ce processus.

L'accessibilité aux professionnels. Certaines répondantes mentionnent ne pas avoir accès à l'équipe interdisciplinaire afin de soutenir l'intervention auprès de l'enfant. D'autres mentionnent que le service est accessible pour leur territoire, mais que l'équipe n'est pas disponible pour recevoir l'enfant en début de services. Cette difficulté à accéder à l'équipe interdisciplinaire est perçue par certaines comme une limite à la portée de leur évaluation initiale, et, par le fait même, à la planification de l'intervention. Elles rapportent se sentir, dans certaines situations, moins bien outillés pour préciser les besoins ou les stratégies à mettre en place (p. ex., lorsqu'un enfant présente des difficultés langagières plus spécifiques).

La multiplication du nombre de personnes présentes lors des rencontres. Pour bon nombre de répondantes, l'un des défis associés au travail en équipe interdisciplinaire en contexte d'ICI est la difficulté à trouver des moments pour réaliser les rencontres. En effet, plus le nombre de participants à l'élaboration du PI est important, plus il est difficile de faire concorder les agendas de toutes les personnes impliquées. Ces difficultés de fixer les moments de rencontres avec l'équipe interdisciplinaire entrainent parfois des délais dans l'élaboration du PI.

À ces difficultés de gestion de l'horaire, s'ajoutent parfois des difficultés concernant l'identification d'objectifs interdisciplinaires. En effet, certaines répondantes mentionnent qu'il peut être difficile, entre les membres de l'équipe, de bien se comprendre et de s'entendre sur les objectifs à prioriser au PI de l'enfant. Il en va de même pour le choix ou la mise en place des stratégies. Il peut y avoir certains écarts entre la façon de concevoir l'intervention selon la discipline. Selon elles, il doit y avoir un effort d'arrimage entre les stratégies proposées et le programme général d'intervention. En fait, certaines répondantes soulignent même que des objectifs au PI sont parfois rattachés de façon très spécifique à la discipline des professionnels impliqués, laissant quelquefois de côté les objectifs plus directement liés au programme, habituellement portés par les TES. Selon quelques répondantes, dans de telles situations, les TES peuvent avoir de la difficulté à appliquer, faire le suivi ou évaluer certains objectifs identifiés au PI.

Pas que des défis. Il faut tout de même souligner que l'accent est ici mis sur les défis, mais pour plusieurs, le travail en équipe interdisciplinaire est perçu comme un important avantage.

«Tu sais, moi je vois juste ça plus facilitant. $C$ 'est tellement le fun maintenant [...], on s'assoit tout de suite avec l'équipe. Je ne vois pas (inspiration), euh, je ne vois pas de défi. Peut-être plus de leur part, parce que ça leur demande beaucoup plus, mais nous autres ça nous apporte tellement. Je ne vois pas vraiment cela comme un défi, là.» (Participant TES 12)

L'une des superviseures mentionne également que les intervenantes acquièrent des connaissances qui sont généralisables à d'autres situations lorsqu'ils participent à l'élaboration du PI en équipe interdisciplinaire, ce travail avec différents professionnels contribuant donc à la formation continue.

«[...] mais par contre, le fait de travailler avec l'équipe multidisciplinaire je trouve ça super intéressant parce qu'on prend les décisions ensemble... plus soutenant que lorsqu'on a à, lorsqu'on fait le processus tout seul... Bien c'est ça le fait d'être bien supporté. » (Participant TES 01) 
Pour faire face aux défis. Le besoin se fait sentir de réfléchir sur les rôles des différents membres de l'équipe interdisciplinaire et de leur implication aux différentes étapes de l'élaboration du PI. Parmi les solutions proposées, certaines concernent plus spécifiquement le soutien clinique, et ce par le biais de différentes modalités (équipe interdisciplinaire, échanges cliniques, supervision). En effet, une répondante propose de prendre le temps de faire des échanges cliniques portant sur les pratiques incluant les TES, mais aussi les autres membres de l'équipe interdisciplinaire. D'ailleurs, les répondantes soulignent le besoin de mieux soutenir le travail interdisciplinaire et proposent des solutions afin de rendre l'équipe plus fonctionnelle, notamment une meilleure planification de l'horaire et une meilleure organisation des rencontres. Il est aussi de faire des rencontres par visioconférences.

\section{L'individualisation en contexte de planification de l'intervention en ICI}

Plusieurs intervenantes évoquent l'individualisation comme étant au cœur de leur travail quotidien, qui est perçue comme essentielle. L'analyse des propos des répondantes concernant l'individualisation de l'intervention en contexte d'ICI permet de constater certains défis liés au choix des objectifs d'intervention et des moyens à mettre en place pour chaque enfant.

Le choix des objectifs. Quelques répondantes mentionnent la difficulté d'élaborer un objectif pour bien répondre aux besoins et aux caractéristiques de chaque enfant. Une répondante souligne cependant qu'avec l'expérience, il devient plus facile de s'éloigner des objectifs préétablis et d'ajuster les objectifs à la programmation en fonction de la réalité de l'enfant. Il est également mentionné que la perspective développementale peut venir soutenir le choix des objectifs à travailler.

Le choix des moyens. Les défis liés à la sélection des moyens les plus appropriés sont rapportés comme étant encore plus importants lorsqu'il s'agit d'enfants ayant des profils de fonctionnement plus extrêmes, soit des enfants dits de haut niveau de fonctionnement ou de faible niveau de fonctionnement. Le manque de formation à utiliser des interventions pour la clientèle présentant des besoins plus importants sur le plan développemental est rapporté comme étant un facteur contribuant à complexifier la sélection des moyens.

" C'est quand on arrive dans les extrêmes des enfants, je pense que ça peut être plus difficile, là, quand on a des [enfants présentant des profils de] très bas niveaux ou des [enfants présentant des profils de] très hauts niveaux que, là, on, ça, il faut sortir un peu de notre cadre et s'ajuster fortement, là » (Participant TES 10)

Pour faire face aux défis. La formation et la supervision ont été mentionnées comme étant nécessaires afin de mieux répondre à la nécessité d'individualiser l'offre de services en fonction du profil de l'enfant, de s'approprier davantage le programme et les stratégies d'intervention ou encore de favoriser un travail d'équipe plus concerté. Les répondantes mentionnent aussi le besoin de formation sur les diverses stratégies et approches associées à l'ICI.

\section{Les bonnes pratiques et les pratiques basées sur les données probantes}

Lorsqu'interrogées sur les défis associés aux données probantes en contexte de planification de l'intervention en ICI, quelques répondantes rapportent qu'il peut être exigeant pour les équipes de maintenir leurs connaissances à jour. En effet, il semble qu'il y ait des défis en termes de temps à consacrer à la formation continue. Pourtant, bon nombre de répondantes soulignent que l'accès aux données issues de la recherche s'avère un atout important dans leur pratique. En effet, malgré les différentes stratégies mises en place par leur organisation (p. ex., les capsules thématiques), quelquesunes des répondantes mentionnent qu'elles arrivent difficilement à mettre à jour leurs connaissances concernant la clientèle et les pratiques à adopter. Des répondantes soulignent que l'accès aux informations (sous forme de consultation, de documents maison ou d'ouvrages) concernant les données de la recherche est relativement limité pour les TES. Les superviseurs et les conseillers au programme jouent un rôle actif dans le transfert de ces connaissances vers l'application sur le terrain par les intervenants. Aussi, une répondante souligne que l'un des défis est l'abondance d'informations et aussi l'accès par les parents à une multitude d'informations à propos des TSA. Il s'avère parfois difficile de s'assurer de la robustesse de celles-ci. Pour certaines répondantes, il peut être difficile d'appliquer les données relevant de la littérature aux interventions sur le terrain, notamment en raison du besoin de les adapter à la réalité terrain, les contextes dans lesquels les études sont réalisées ou les profils des enfants pouvant différer de ce qu'ils rencontrent en contexte réel d'intervention. 
Le processus clinique de l'établissement mis en place par l'établissement

L'analyse des entrevues permet de constater certains défis associés au processus clinique, notamment en lien avec : 1) le niveau de familiarisation avec le processus; 2) le nombre restreint d'objectifs au PI; 3) les délais accordés pour le premier PI; 4) le processus de révision du PI et de la programmation; 5) les tâches cléricales associées au processus; et 6) l'arrimage entre le programme et un autre cadre conceptuel, soit celui du processus de production du handicap ( $\mathrm{PPH}$; Fougeyrollas, 2010; Fougeyrollas, Cloutier, Bergeron, Côté et StMichel, 1998).

La familiarisation avec le processus clinique. Certaines répondantes relèvent des difficultés à se familiariser avec le processus clinique, en raison notamment du faible nombre de PI pour lesquels les TES sont appelés à contribuer sur une base annuelle. De façon plus spécifique, des répondantes soulignent les difficultés liées à l'élaboration d'objectifs répondant aux critères de qualite établis par le processus clinique. De plus, puisque la majorité des intervenantes n'élaborent qu'un PI annuellement, les chances qu'il y ait eu des modifications au processus clinique entre deux processus d'élaboration de PI ajoutent à la difficulté d'appropriation. Ce constat est le même pour une partie de l'équipe interdisciplinaire.

Le nombre d'objectifs au PI. Le nombre limité d'objectifs qu'il est permis d'inscrire au PI, en raison de règles administratives et de contraintes de logiciel, semble être une contrainte pour plusieurs. Bien qu'elles partagent le souci d'éviter que le processus de planification ne se traduise par un document qui soit trop lourd, plusieurs répondantes estiment que le PI reflète mal l'ensemble du travail réalisé auprès de l'enfant. Pour plusieurs répondantes, la programmation est un meilleur reflet de ce qui est réellement travaillé avec l'enfant. Cependant, il peut exister des difficultés d'arrimage entre la programmation et le PI. Les répondantes mentionnent qu'il peut être complexe de faire en sorte que les objectifs travaillés dans le cadre de la programmation soient en lien avec ceux identifiés au PI.

Les délais accordés pour réaliser le premier PI. Les répondantes mentionnent que le délai entre l'entrée en services et l'élaboration du premier PI est court. Elles évoquent notamment les retards occasionnés en raison des défis associés aux rencontres avec l'équipe interdisciplinaire et les parents. Par manque de temps, certaines étapes sont parfois allégées, notamment celle de la cueillette de données.

La révision du plan d'intervention et de la programmation. Les répondantes rapportent des difficultés associées à des révisions de PI qui ne suivent pas le rythme de l'enfant.

«En ICI, qu'est-ce qui est difficile c'est de, que l'enfant évolue des fois plus vite que le PI peut nous le permettre de changer nos choses [...] c'est parce que, quand tu fais l'évaluation au bout de trois mois puis que cela fait un mois que c'est déjà acquis. » (Participant TES 07)

L'arrimage avec un autre cadre conceptuel. Les services de réadaptation du réseau de la santé ont opté pour un modèle conceptuel général auquel les intervenants doivent se référer lors de l'élaboration des PI, soit le PPH. Des répondantes soulignent la difficulté de l'arrimage de ce modèle conceptuel avec le contexte d'ICI avec de jeunes enfants. Certaines rapportent des difficultés à s'approprier ce modèle conceptuel.

"Puis surtout dans l'optique de l'arrimage aussi notre défi c'est l'arrimage avec le PPH qui est très, euh. Je trouve cela génial le PPH, mais au niveau du zéro-cinq ans, toute la sphère développementale dans le PPH. On travaille fort pour essayer de rentrer cela làdedans en se disant bien on prévient des situations handicaps. »

«On a tout le volet développemental, parce que dans le fond, en 0-5 ans, on travaille beaucoup en prévention ou en, on travaille beaucoup sur le plan développement, on veut que l'enfant évolue. Et donc, en travaillant sur le développement de l'enfant bien la situation de handicap est pas toujours là. »

Pour faire face aux défis. L'ICI diffère, à certains égards, des autres offres de services de l'établissement. Ainsi, certaines répondantes soulignent le besoin de bien clarifier l'articulation du processus clinique dans le contexte spécifique de l'ICI, notamment en précisant l'arrimage possible avec le PPH. En ce sens, il est proposé de créer un canevas en soutien à l'élaboration des PI et un aide-mémoire afin de permettre à la TES ou à l'ensemble de l'équipe d'autoévaluer leur propre processus de planification de l'intervention.

Quelques répondantes mentionnent le besoin d'outils utiles dans la formulation adéquate d'objectifs selon les critères de qualité établis par l'établissement. Pour certains, un tel outil permettrait également une économie 
de temps. Quelques répondantes proposent d'élaborer une liste d'objectifs déjà formulés correspondant aux besoins les plus souvent rencontrés en ICI que la TES pourrait utiliser en l'adaptant aux spécificités de l'enfant. Sans être une liste d'objectifs à proprement dit, les répondantes proposent que soient rendus disponibles des exemples d'objectifs opérationnels et ce, à l'ensemble de l'équipe interdisciplinaire afin de favoriser la cohérence. D'autres entrevoient la possibilité d'une formation ou de lieux d'échanges et de discussions cliniques en y associant le besoin d'une compréhension commune entre les membres de l'équipe. Au-delà de la formulation proprement dite, une des professionnelles mentionne le besoin de revoir la procédure de sélection et de priorisation des objectifs.

\section{Discussion}

Cette étude visait à identifier et mieux comprendre les défis associés à l'élaboration des PI en contexte d'ICI tels que perçus par les membres des équipes d'intervention. Des entrevues ont été réalisées auprès de 31 membres d'équipes d'intervention en ICI recrutés dans un établissement québécois. L'analyse de contenu réalisée permet de mettre en lumière différents défis que des intervenantes rapportent rencontrer dans le cadre de ce processus. Aucune répondante n'a décrit la démarche d'élaboration des PI comme étant simple ou facile. Cela vient corroborer les écrits soulignant la complexité d'une telle démarche (Leaf et al., 2016). Il est possible de regrouper les défis selon deux grandes dimensions, soit 1) les défis cliniques et 2 ) les défis d'encadrement et de soutien à la pratique.

Sur le plan clinique. L'un des premiers constats est certainement la reconnaissance, par les personnes qui implantent l'ICI, de l'importance de l'individualisation de l'intervention offerte aux jeunes enfants ayant un TSA et donc d'une bonne planification de celle-ci. Cet aspect rejoint l'idée que l'individualisation est un aspect central d'une ICI de qualité (Leaf et al., 2016; Leaf et McEachin, 2016; McCollow et Hoffman, 2018; Romanczyk et Gillis, 2011). Leaf et McEachin (2016) opposent d'ailleurs ce constat à la croyance populaire voulant que l'ICI consiste en une application rigide d'un protocole unique. Les propos mettent également l'emphase sur le processus d'élaboration des PI. Il est l'outil qui rend compte de ce processus d'individualisation en y précisant les objectifs et les moyens à mettre en place pour l'enfant recevant les services, tel que prévu par la Loi sur les services de santé et les services sociaux (LSSS).
La fonction de planification de l'intervention. Plusieurs éléments rapportés comme constituant des défis sont associés à la fonction première du PI, celle de planification de l'intervention (Goupil, 2004). Les répondantes mentionnent qu'il peut être complexe d'identifier les objectifs et les moyens à mettre en place pour chaque enfant. Cela étant cohérent avec les propos de Tarbox et al. (2013) spécifiant que l'élaboration d'un PI pour les jeunes enfants ayant un TSA est donc un processus complexe et multidimensionnel, qui tient compte d'un ensemble de facteurs. Afin de les soutenir en ce sens, elles mentionnent le besoin de mieux connaitre les différents curriculums ou modèles d'ICI. Cette recherche de modèle semble liée au besoin d'individualisation de l'intervention. En effet, les intervenantes cherchent des documents afin de les soutenir dans le processus d'identification des cibles d'intervention pour les enfants. Le curriculum le plus répandu, soit celui de Lovaas (1996) n'est pas suffisant selon les répondantes. Le recours à la combinaison de différents curriculums dans le cadre de l'ICI par les intervenants des établissements est d'ailleurs documenté dans le cadre d'une étude provinciale (Dionne et al., 2016). De plus, le désir d'accéder aux curriculums des différents modèles est possiblement associé au besoin de clarifier le modèle d'intervention privilégié par les établissements, en précisant notamment la façon dont les équipes d'intervention doivent procéder dans l'identification des buts, des objectifs et des stratégies à mettre en place pour un enfant. C'est d'ailleurs un autre constat de l'étude sur l'implantation de l'ICI au Québec, peu d'établissements possèdent une documentation claire concernant leur modèle d'intervention (Dionne et al., 2016). Or, les curriculums constituent un soutien aux équipes d'intervention en précisant ce qui est enseigné et comment l'enseigner, ce qui inclut l'identification des buts, des objectifs, des stratégies d'intervention, de même que des outils ou moyens d'évaluation (Chiang, 2017). Ils servent donc de ligne directrice aux équipes cliniques.

D'ailleurs, une clarification du modèle d'intervention, de même que la formation à l'ensemble de l'équipe peuvent s'avérer être des occasions de soutenir le travail interdisciplinaire en favorisant une compréhension commune (Cox, 2012). En effet, les intervenants soulignent le besoin d'une formation commune à l'ensemble de l'équipe clinique, permettant un langage commun, mais aussi une clarification des rôles de chacun. Ceci fait référence à la notion de partage des responsabilités du PI selon Goupil (2004). 
À cet effet, les répondantes soulignent que bon nombre d'enfants reçoivent l'ICI en milieu de garde ou à domicile, contrairement à certains modèles qui offrent l'intervention en clinique. Cela nécessite d'avoir recours à des stratégies d'intervention compatibles avec ces milieux de vie. Évidemment, il est possible de planifier une intervention en individuel dans un local séparé, ce qui comporte certains avantages. Cependant, une offre d'ICI en milieu naturel permet l'accès à des occasions d'apprentissages contextualisés (Leaf, Leaf, McEachin, Cihon et Ferguson, 2018). Afin de planifier ces occasions, il est nécessaire pour l'équipe de considérer les points de vue et réalités des partenaires de ces milieux. Cela renvoie d'ailleurs à la notion de validité sociale, principe au cœur de l'analyse appliquée du comportement selon lequel une intervention n'est valide que si ses objectifs sont jugés pertinents, ses procédures acceptables et ses résultats satisfaisants pour la société (Clément et Schaeffer, 2010; Paquet, McKinnon, Clément et Rousseau, 2018). Il s'avère donc nécessaire que les intervenants planifient l'intervention de façon concertée avec non seulement la famille, mais également les milieux que l'enfant fréquente et/ou une partie de l'intervention se déroule. Cela réfère à la fonction de concertation du PI, tel que suggéré par Goupil (2004).

La notion d'intensité de l'intervention, entraînant la poursuite d'un grand nombre d'objectifs d'intervention, est également un élément soulevé comme étant un défi par un grand nombre de répondantes. Bien que perçue comme étant un des avantages de l'ICI, l'intensité impose une certaine pression en termes de planification et de partage des informations sur l'intervention avec les différents acteurs. En effet, les répondantes mentionnent le grand nombre d'objectifs poursuivis à la fois, mais également la rapidité avec laquelle ces objectifs peuvent être atteints et ainsi, la nécessité de les revoir sur une base régulière. Selon les propos recueillis, la lourdeur administrative et technique du processus d'élaboration des PI peut être difficilement conciliable avec cette intensité. En effet, les établissements, par le biais de leur processus clinique et selon les exigences légales, prévoient habituellement une révision des objectifs au PI selon un intervalle de trois mois (CRDITED MCQ - IU, 2013). Selon les propos recueillis, la révision des objectifs d'intervention est réalisée beaucoup plus fréquemment en contexte ICI. Les répondantes suggèrent que la programmation individuelle soit une façon de répondre à ce défi de planification d'un grand nombre d'objectifs, celle-ci étant annexée au PI. Par contre, elles mentionnent également l'importance que cette programmation individuelle soit en lien très étroit avec le PI, non pas une annexe indépendante. Dans un tel contexte, le PI ne répondrait plus à ses fonctions premières, soit celles de témoigner de la planification, de la gestion, de la concertation, tout en étant un outil de communication (Goupil, 2004). Les propos recueillis permettent de constater que les répondants sont à la recherche d'une solution, d'un équilibre, permettant au PI de conserver ses fonctions premières, soulignant au passage l'importance de la communication avec la famille.

Sur le plan de l'encadrement. Les intervenantes mentionnent aussi des défis d'appropriation du processus clinique en raison du petit nombre d'enfants qu'elles côtoient et ainsi, le petit nombre de PI auxquels elles participent. À cet effet, Leaf et al. (2018) notent que lorsque les intervenants interviennent auprès d'un petit nombre d'enfants à la fois, ils mettent plus de temps à gagner en expérience. Cela signifie qu'ils ont besoin davantage d'accompagnement. À cet effet, malgré les besoins de formation et de soutien clinique relevés par les répondants et par la littérature (Eikeseth, 2010; Leaf et al., 2016), à notre connaissance, il n'existe pas de cadre venant clarifier les modalités de soutien clinique, notamment la formation, la supervision et le coaching, spécifique au contexte de services ICI dans les établissements québécois. Des balises doivent donc être développées. D'ailleurs plusieurs études portent sur la supervision en contexte d'ICI (Eikeseth, 2010; Eikeseth, Hayward, Gale, Gitlesen et Eldevk, 2009; Gibson, Grey et Hastings, 2009; Grey, Honan, McClean et Daly, 2005; Leblanc et Luiselli, 2016; Paquet et al., 2017; Symes, Remington, Brown et Hastings, 2006).

En ce qui concerne le soutien professionnel, les répondantes mentionnent également un besoin de soutien dans le rôle qu'elles ont à jouer auprès des parents afin de favoriser leur participation à chacune des étapes de la planification de l'intervention (Bradshaw et al., 2017; Ogilvie et McCrudden, 2017). En effet, il ressort assez clairement que, lors de la planification de l'intervention, les intervenantes sont appelées à réaliser différents types d'intervention auprès des familles pour lesquels elles ne se sentent pas toujours à l'aise. Qu'il s'agisse de soutenir les parents lors de l'identification des besoins de leur enfant, relativement à l'adaptation face au diagnostic, lors du partage d'information relative à l'intervention, lors de la transmission et du partage d'informations relatives aux progrès de l'enfant, ce soutien reste un défi pour les répondantes. Cela rejoint les résultats de l'étude d'Abouzeid et Poirier (2014) relevant que la nécessité 
d'améliorer le soutien offert aux familles, tant sur le plan social, émotionnel, informatif qu'instrumental est présente, car les intervenantes et les superviseures sont une importante source de soutien pour les mères d'enfants ayant un TSA en contexte d'ICI.

\section{Conclusion}

Certaines limites de l'étude doivent être prises en compte dans l'interprétation des résultats de cette étude. En effet, les résultats reposent sur des propos recueillis et n'ont pas été triangulés avec des observations ou des données administratives. Aussi, les participantes ont été recrutées au sein d'un seul établissement. Cela a pour effet d'augmenter la compréhension du phénomène étudié au sein même de l'établissement. Cependant, cela affecte la transférabilité des résultats à d'autres contextes. De plus, les résultats reflètent les perceptions au moment de l'étude. Ces perceptions, tout comme les pratiques sont susceptibles de se transformer dans le temps.

Malgré les limites énoncées, certains aspects méthodologiques viennent soutenir l'intérêt des résultats présentés. Le taux de participation élevé au sein d'une seule équipe permet une représentativité des propos et perceptions rapportés. De plus, cette étude permet de mettre l'accent sur une réalité bien particulière, soit celle de la planification du programme ICI dans un contexte d'offre de services à large échelle dans la communauté par un établissement public. Peu d'informations étaient disponibles concernant les défis particuliers qu'entraine la planification de l'intervention à offrir dans ce contexte particulier. Cette étude s'inscrit dans une longue démarche de collaboration recherche-pratique afin de mieux cerner la réalité des milieux d'intervention afin de permettre des innovations pouvant répondre aux besoins des intervenants en contexte réel d'intervention. Cette collaboration étroite recherche-pratique est d'ailleurs proposée comme une des solutions prometteuses dans la tentative de pallier les difficultés de mise en place des pratiques basées sur les données probantes en contexte réel d'intervention (Parsons et al., 2013).

Évidemment, il s'agit d'une première étape d'exploration des défis associés à la planification de l'intervention. Beaucoup reste à faire afin de mieux comprendre les différents enjeux relatifs à la planification de l'intervention individualisée pour les jeunes enfants ayant un TSA et ainsi pouvoir mieux soutenir les intervenants dans leur rôle. Cette étude laisse entrevoir plusieurs pistes de recherche sur des variables pouvant influer sur la qualité de l'ICI offerte, notamment la question de la collaboration et la participation des familles. Ainsi, il est proposé de mieux outiller les intervenants au travail de soutien et de collaboration. Pour ce faire, il est possible de prendre appui sur les bonnes pratiques en intervention précoce recommandant de prendre appui sur une approche centrée sur la famille (DEC, 2014). Aussi, les résultats viennent réaffirmer le besoin de clarifier plusieurs aspects du protocole d'intervention, tels qu'énoncés dans le cadre d'une enquête provinciale (Dionne et al., 2016), notamment le rôle des différents intervenants et professionnels et les modalités de soutien à la pratique. Il nous apparait également nécessaire de revoir la façon dont sont ciblés les objectifs afin d'assurer un lien étroit entre le travail réalisé et le suivi des progrès au quotidien et le plan d'intervention. Pour ce faire, une identification des cibles d'intervention, prenant appuis sur un modèle développemental comprenant des objectifs spécifiques et des buts hiérarchisés, est une avenue à explorer. Certains modèles d'intervention (p. ex. le DATA Model de Schwartz et al., 2017) ont fait le choix d'utiliser un outil d'évaluation développemental permettant de lier l'évaluation aux cibles d'intervention du PI, à l'aide d'objectifs spécifiques et de grands buts (le AEPS, Bricker, 2002). Le recours à un tel outil a d'ailleurs été privilégié dans le cadre d'un projet de développement de protocole réalisé en collaboration avec sept établissements québécois (pour une description plus complète du projet, voir Dionne, Paquet et Boutet, Sous presse). Cette démarche d'amélioration des pratiques d'intervention comportementale intensive par un partenariat étroit entre recherche et pratique avait notamment pour visée une réponse aux défis de réduire les écarts entre les connaissances issues de la recherche et leur application à un contexte réel d'intervention. Des suites à donner sont certainement d'évaluer l'impact du recours à de tels instruments d'évaluation sur les pratiques d'élaboration des PI, les avantages de leur utilisation et les défis qui persistent. 


\title{
INDIVIDUALIZED EDUCATION PLAN IN EARLY INTENSIVE BEHAVIORAL INTERVENTION (EIBI): PERCEPTION OF PRACTICES AND CHALLENGES
}

\begin{abstract}
The application of Early Intensive Behavior Intervention (EIBI) requires elaborating objectives and strategies for each child. This individualization process is essential for a quality intervention. By law, institutions are accountable for the planning of interventions for each person to whom they provide services through the intervention plan. This study aims to identify the practices and challenges associated with developing these plans, within EIBI, as perceived by members of the intervention team (21 caseworkers, 6 supervisors, and 4 professionals). Analysis of semi-structured interviews helps identify perceived challenges, including those associated with intervention characteristics, parental involvement, interdisciplinary work, individualization, access to evidence-based data to support intervention planning and the standards of the institution.
\end{abstract}

Keywords: Early intensive behavioral intervention, autism, intervention plan, community, practitioner's perceptions.

\section{Références}

Abouzeid, N. et Poirier, N. (2014). Perceptions de mères d'enfant présentant un trouble du spectre de l'autisme ayant reçu une intervention comportementale intensive : leurs ressources et leurs défis. Revue de psychoéducation, 43(2), 201-233.

Baer, D. M., Wolf, M. M. et Risley, T. R. (1968). Some current dimensions of applied behavior analysis. Journal of Applied Behavior Analysis, 1, 91-97.

Boutin, G. (2018). L'entretien de recherche qualitatif $\left(2^{\mathrm{e}}\right.$ éd.). Québec, QC: Presses de l'Université du Québec.

Bradshaw, J., Bearss, K., McCracken, C., Smith, T., Johnson, C., Lecavalier, L., ... Scahill, L. (2017). Parent education for young children with autism and disruptive behavior: Response to active control treatment. Journal Clinical Child Adolescent Psychological, 19, 1-11. doi:10.1080/15374416.2017.1381913

Bricker, D. (2002). Assessment evaluation and programming system for infants and children ( $2^{\mathrm{e}}$ éd.). Baltimore, MD: Paul H. Brookes.

Centre de réadaptation en déficience intellectuelle et en trouble envahissants du développement de la Mauricie et du Centre-du-Québec - Institut universitaire. (2013). Processus clinique. Trois-Rivières, QC: Auteur.

Chiang, H.-M. (2017). Foundations and development of curriculum. Dans H.-M. Chiang (dir.), Curricula for teaching students with autism spectrum disorder (p. 1-19). Cham, Suisse: Springer.

Clément, C. et Schaeffer, E. (2010). Évaluation de la validité sociale des interventions menées auprès des enfants et adolescents avec un TED. Revue de psychoéducation, 39(2), 207-218.
Cox, D. J. (2012). From interdisciplinary to integrated care of the child with autism: The essential role for a code of ethics. Journal of Autism and Developmental Disorders, 42(12), 2729-2738. doi:10.1007/s10803012-1530-z

Dingfelder, H. E. et Mandell, D. S. (2011). Bridging the research-to-practice gap in autism intervention: An application of diffusion of innovation theory. Journal of Autism and Developmental Disorders, 41(5), 597609. doi:10.1007/s10803-010-1081-0

Dionne, C., Joly, J., Paquet, A., Rousseau, M. et Rivard, M. (2016). Organizations' choices when implementing an early intensive behavioral intervention program (EIBI). Education Sciences \& Society, 7(2), 150-171.

Dionne, C., Paquet, A. et Boutet, M. (Sous presse). Démarche d'amélioration des pratiques d'intervention intensive par un partenariat recherche-pratique : concilier meilleures pratiques et transférabilité en situation réelle. Approche neuropsychologique des apprentissages chez l'enfant, 162.

Division for Early Childhood. (2014). DEC recommended practices in early intervention/early childhood special education 2014. Repéré à http://www.dec-sped.org/recommendedpractices

Eikeseth, S. (2010). Examination of qualifications required of an EIBI professional. European Journal of Behavior Analysis, 11(2), 239-246. doi:10.1080/15021149.2010.114348

Eikeseth, S., Hayward, D., Gale, C., Gitlesen, J.-P. et Eldevik, S. (2009). Intensity of supervision and outcome for preschool-aged children receiving early intensive behavioral interventions: A preliminary study. Research in Autism Spectrum Disorders, 3(1), 67-73. doi:10.1016/j.rasd.2008.04.003 
Eldevik, S., Hastings, R. P., Hughes, J. C., Jahr, E., Eikeseth, S. et Cross, S. (2010). Using participant data to extend the evidence base for intensive behavioral intervention for children with autism. American Journal on Intellectual and Developmental Disabilities, 115(5), 381-405.

Fougeyrollas, P. (2010). La funambule, le fil et la toile: transformations réciproques $d u$ sens $d u$ handicap. Québec, QC: Presses de l’Université Laval.

Fougeyrollas, P., Cloutier, R., Bergeron, H., Côté, J. et StMichel, G. (1998). Classification québécoise. Processus de production du handicap. Québec, QC: RIPPH/SCCIDIH.

Gibson, J. A., Grey, I. M. et Hastings, R. P. (2009). Supervisor support as a predictor of burnout and therapeutic self-efficacy in therapists working in ABA schools. Journal of Autism and Developmental Disorders, 39, 1024-1030.

Goupil, G. (2004). Plans d'intervention, de services et de transition. Montréal, QC: Gaétan Morin.

Grey, I. M., Honan, R., McClean, B. et Daly, M. (2005). Evaluating the effectiveness of teacher training in applied behaviour analysis. Journal of Intellectual Disabilities, 9(3), 209-227.

Haute Autorité de santé. (2012). Autisme et autres troubles envahissants $d u$ développement : interventions éducatives et thérapeutiques coordonnées chez l'enfant et l'adolescent. Recommandation de bonne pratique. Repéré à https://www.has-sante.fr/portail/upload/do cs/application/pdf/2012-03/recommandations_autism e_ted_enfant_adolescent_interventions.pdf

Institut national d'excellence en santé et en services sociaux. (2014). L'efficacité des interventions de réadaptation et des traitements pharmacologiques pour les enfants de 2 à 12 ans ayant un trouble du spectre de l'autisme (TSA) : édition révisée. Évaluation des technologies et modes d'intervention en santé, 10(3), 1-67.

Kasari, C. et Lawton, K. (2010). New directions in behavioral treatment of autism spectrum disorders. Current Opinion in Neurology, 23(2), 137-143. doi:10.1097/WCO.0b013e328 33775cd

Klintwall, L. et Eikeseth, S. (2014). Early and intensive behavioral intervention (eibi) in autism. Dans V. B. Patel, V. R. Preedy et C. R. Martin (dir.), The comprehensive guide to autism (p. 117-137). New York, NY: Springer.

Kuppens, S. et Onghena, P. (2012). Sequential metaanalysis to determine the sufficiency of cumulative knowledge: The case of early intensive behavioral interventions for children with autism spectrum disorders. Research in Autism Spectrum Disorders, 6, 168-176.
Långh, U., Cauvet, E., Hammar, M. et Bölte, S. (2017). Cross-cultural validation of the york measure of quality of intensive behavioral intervention. Behavior Modification, 41(6), 808-828. doi:10.1177/01454455 17719397

Leaf, J. B., Leaf, R., McEachin, J., Cihon, J. H. et Ferguson, J. L. (2018). Advantages and challenges of a hom- and clinic-based model of behavioral intervention for individuals diagnosed with autism spectrum disorder. Journal of Autism and Developmental disorders, 48(6), 2258-2266. doi:10.1007/s10803-017-3443-3

Leaf, J. B., Leaf, R. McEachin, J., Taubman, M., Ala'iRosales, S., Ross, R. K. et Weiss, M. J. (2016). Applied behavior analysis is a science and, therefore, progressive. Journal of Autism and Developmental Disorders, 46(2), 720-731. doi: 10.1007/s10803-0152591-6

Leaf, R. et McEachin, J. (1999). A work in progress. New York, NY: Different Roads to Learning, inc.

Leaf, R. et McEachin, J. (2016). The Lovaas model: Love it or hate it, but first understand it. Dans R. G. Romanczyk et J. McEachin (dir.), Comprehensive models of autism spectrum disorder treatment (p. 743). Cham, Suisse: Springer International Publishing.

Leblanc, L. A. et Luiselli, J. K. (2016). Refining supervisory practices in the field of behavior analysis: Introduction to the special section on supervision. Behavior Analysis in Practice, 9(4), 271-273. doi:10.1007/s40617-016-0156-6

Lovaas, O. I. (1996). The UCLA young autism model of service delivery. Dans C. Maurice, G. Green et S. C. Luce (dir.), Behavioral intervention for young children with autism: A manual for parents and professionals (pp. 241-248). Austin, TX: PRO-ED.

Makrygianni, M. K., Gena, A., Galanis, P. et Katoudi, S. (2018). The effectiveness of applied behavior analytic interventions for children with Autism Spectrum Disorder: A meta-analytic study. Research in Autism Spectrum Disorder, 51, 18-31. doi:10.1016/j.rasd.2 018.03 .006

Makrygianni. M. K. et Reed, P. (2010). A meta-analytic review of the effectiveness of behavioural early intervention programs for children with autism spectrum disorders. Research in Autism Spectrum Disorders, 4(4), 577-593. doi:10.1016/j.rasd.2010.01.014

Masi, A., DeMayo, M. M., Glozier, N. et Guastella, A. J. (2017). An overview of autism spectrum disorder, heterogeneity and treatment options. Neuroscience Bulletin, 33(2), 183-193. doi:10.1007/s12264-017-0100-y

Maurice, C., Green., G. et Luce, S. C. (1996). Behavioral intervention for young children with autism: A manual for parents and professionals. Austin, TX: PRO-ED. 
McCollow, M. M. et Hoffman, H. H. (2018). Evidencebased decision-making: A team effort toward achieving goals. Young Exceptional Children, 1-9. doi:10.1177/109625061 8801287

McGee, G. G., Morrier, M. J. et Daly, T. (1999). An incidental teaching approach to early intervention for toddlers with autism. Journal of the Association of Persons with Severe Handicaps, 24, 133-146. doi:10.2511/rpsd.24.3.133

ministère de la Santé et des Services sociaux. (1972). Loi sur les services de santé et les services sociaux. Québec, QC: Auteur.

Ministère de la Santé et des Services sociaux. (2003). Un geste porteur d'avenir : des services aux personnes présentant un trouble envahissant de développement, à leurs familles et leurs proches. Québec, QC: Auteur

Odom, S. L., Boyd, B. A., Hall, L. J. et Hume, K. (2010). Evaluation of comprehensive treatment models for individuals with autism spectrum disorders. Journal of Autism and Developmental Disorders, 40(4), 425436. doi:10.1007/s10803-009-0825-1

Odom, S. L., Collet-Klingenberg, L., Rogers, S. J. et Hatton, D. D. (2010). Evidence-based practices in interventions for children and youth with Autism Spectrum Disorders. Preventing School Failure: Alternative Education for Children and Youth, 54(4), 275-282. doi:10.1080/10459881003785506

Ogilvie, E. et McCrudden, M. T. (2017). Evaluating the social validity of the early start Denver model: A convergent mixed methods study. Journal of Autism and Developmental Disorders, 47(9), 2899-2910. doi:10.1007/s10803-017-3214-1

Paillé, P. et Mucchielli, A. (2016). L'analyse quantitative en sciences humaines et sociales. Paris, France: Armand Colin.

Paquet, A., Dionne, C., Joly, J., Rousseau, M. et Rivard, M. (2017). Supervision of large-scale communitybased early intensive behavioral intervention programs in Quebec: Description of practices. Journal on Developmental Disabilities, 23(1), 54-63.

Paquet, A., McKinnon, S., Clément, C. et Rousseau, M. (2018). Traduction et adaptation du TEI-SF afin de documenter l'acceptabilité sociale de l'intervention comportementale intensive. Pratiques psychologiques, 24(1), 99-113. doi:10.1016/j.prps.2017.01.004

Parsons, S., Charman, T., Faulkner, R., Ragan, J., Wallace, S. et Wittemeyer, K. (2013). Commentary Bridging the research and practice gap in autism: The importance of creating research partnerships with schools. Autism, 17(3), 268-280. doi:10.1177/13623613123 72068
Prior, M., Roberts, J. M. A., Rodger, S., Williams, K. et Sutherland, R. (2011). A review of the research to identify the most effective models of practice in early intervention of children with autism spectrum disorders. Repéré à https://www.dss.gov.au/sites/def ault/files/documents/10_2014/review_of_the_researc h_report_2011_0.pdf

Reichow, B., Hume, K., Barton, E. E. et Boyd, B. A. (2018). Early intensive behavioral intervention (EIBI) for young children with autism spectrum disorders (ASD). Cochrane Database of Systematic Reviews, 5, 1465-1858. doi: 10.1002/14651858.CD009260.pub3

Reichow, B., Barton, E. E., Boyd, B. A. et Hume, K. (2012). Early intensive behavioral intervention (EIBI) for young children with autism spectrum disorders (ASD). Cochrane Systematic Review, 10, 1-63. doi:10.1002/14651858.CD009260.pub2

Romanczyk, R. G. et Gillis, J. M. (2011). Continuumbased model of behavioral treatment for children with autism: A multi-factor and multi-dimensional perspective. Dans E. A. Mayville et J. A. Mulick (dir.), Behavioral foundations of effective autism treatment (p. 201-216). New York, NY : Sloan.

Rousseau, M., Dionne, C. et Paquet, A. (2012). Intervention précoce et instruments d'évaluation des jeunes enfants ayant un trouble du spectre autistique. Revue francophone de la déficience intellectuelle, 23, 93-103. doi:10.7202/1012991ar

Schwartz, I., Ashmun, J., McBride, B., Scott, C. et Sandall, S. (2017). The DATA (Developmentally appropriate treatment for autism) Model for teaching Preschoolers with autism. Maryland, MD: Paul H. Brookes.

Schwartz, I., Sandall, S., McBride, B and Boulware, G. (2004). Project DATA (Developmentally Appropriate Treatment for Autism): An inclusive school-based approach to educating young children with autism. Topics in Early Childhood Special Education, 24 (3), 156-168. doi: 10.1177/02711214040240030301

Strain, P. S. et Bovey, E. H. (2011). Randomized, controlled trial of the LEAP Model for early intervention for young children with autism spectrum disorders. Topics in Early Childhood Special Education, 31(3), 133-154.

Strauss, K., Benvenuto, A., Battan, B., Siracusano, M., Terribili, M., Curatolo, P. et Fava, L. (2015). Promoting shared decision making to strengthen outcome of young children with autism spectrum disorders: The role of staff competence. Research in Developmental Disabilities, 38, 48-63. doi:10.1016/j.ridd.2014.11.016 
Symes, M. D., Remington, B., Brown, T. et Hastings, R. P. (2006). Early intensive behavioral intervention for children with autism: Therapists' perspectives on achieving procedural fidelity. Research in Developmental Disabilities, 27(1), 30-42. doi:10.1016/j.ridd.2004.0 7.007

Tarbox, J., Najdowski, A. C., Bergstrom, R., Wilke, A., Bishop, M., Kenzer, A. et Dixon, D. (2013). Randomized evaluation of a web-based tool for designing function-based behavioral intervention plans. Research in Autism Spectrum Disorders, 7(12), 1509-1517. doi:10.1016/j.rasd.2013.08.005

Virués-Ortega, J. (2010). Applied behavior analytic intervention for autism in early childhood: Metaanalysis, meta-regression and dose-response metaanalysis of multiple outcomes. Clinical Psychology Review, 30(4), 387-399. doi:10.1016/j.cpr.2010.01.008
Warren, Z., McPheeters, M. L., Sathe, N., Foss-Feig, J. H., Glasser, A. et Veenstra-Vanderweele, J. (2011). A systematic review of early intensive intervention for autism spectrum disorders. Pediatrics, 127(5), 13031311. doi:10.1542/peds.2011-0426

Weitlauf, A. S., McPheeters, M. L., Peters, B., Sathe, N., Travis, R., Aiello, R., ... Warren, Z. (2014). Therapies for children with autism spectrum disorder: Behavioral interventions update. Comparative Effectiveness Review, 137, 1-519. 